\title{
Evaluation of the Rapid Card Test and Capture ELISA Tests in Diagnosis of Dengue Infection
}

\author{
Rameena Anver, Vimalkumar Karnaker* and Rekha Rai \\ Department of Microbiology, KS Hegde Medical Academy \\ Deralakatte, Mangalore-575018, Karnataka, India \\ *Corresponding author
}

\begin{tabular}{|l}
\hline K e y w o r d s \\
Dengue, NS1Ag, \\
IgM, IgG, ELISA \\
\hline Article Info \\
\hline $\begin{array}{l}\text { Accepted: } \\
\text { 28 June } 2018 \\
\text { Available Online: } \\
\text { 10 July } 2018\end{array}$ \\
\hline
\end{tabular}

\section{Keywords}

Dengue, NS1Ag,

Article Info

Accepted:

Available Online:

July 2018

\section{A B S T R A C T}

Dengue Fever is a Flaviviral infection transmitted by the Aedes mosquitoes. It is a major public health issue in tropical and subtropical countries. Dengue virus is having four serotypes. It may progress to Dengue Hemorrhagic Fever, which can lead to Dengue Shock Syndrome and death. The study was conducted in the Department of Microbiology. K S. Hegde Medical Academy with the blood samples collected from the patients with clinical diagnoses of Dengue without any age or gender restriction. The samples were collected from the period of October 2015 to April 2017. Two blood samples were collected from each patient. First sample collected between 1-5 days of appearance of clinical symptoms and second sample between 15-21 days. 67 samples were collected and tested with Rapid test (Dengue Day 1Test) and ELISA test for the detection of NS1Ag, IgM and IgG antibody. Results from both methods were compared for evaluation considering Elisa as a gold standard. 67 samples from Dengue positive patients were collected in the time period of study and processed. In first sample, Rapid test showed 54 NSI Ag positive cases, $13 \mathrm{NS} 1 \mathrm{Ag}$ negative, $9 \mathrm{IgM}$ positive, $10 \mathrm{IgG}$ positive and 1 case positive for both IgM and IgG. In ELISA test, no positive cases for IgM and IgG together, but other reports were same. In second sample out of 67 samples 41 were positive for NS1Ag, 26 were negative. IgM positive in 27, IgG positive in 21 and both $\operatorname{IgM}$ and $\operatorname{IgG}$ positive in 9. In Elisa tests NS1Ag positive in 40 negative in 27 . IgM positive in 31 and IgG positive in 25 . Both $\operatorname{IgM}$ and $\operatorname{IgG}$ was positive in 17 cases. Statistical analysis and Evaluation of kits was done considering ELISA as gold standard comparing sensitivity, specificity, positive predictive value, negative predictive value and diagnostic accuracy. Dengue infection is a vector borne disease and it has to be diagnosed early so that control measures can be taken at the earliest to prevent epidemics. Accurate and early diagnoses will decrease the mortality by giving adequate medical care. In a developing country like India most of the hospitals are not well equipped and resource setups are not standardized. So the diagnostic strategy should be based on accuracy and cost effectiveness without causing heavy financial burden to the community and Government. 


\section{Introduction}

Dengue is one of the most rapidly spreading arthropod borne viral disease, which is becoming a major public health problem in tropical and subtropical regions. Dengue virus belongs to family Flaviviridae and it is a positive sense single stranded RNA $(\text { ssRNA }+)^{1}$.

Dengue virus has got four serotypes (DENV1,DEN-2,DEN-3,DENV-4). Dengue is transmitted by the bite of infected female mosquitoes of the genus Aedes aegypti and also Aedes albopictus. This disease causes varying clinical symptoms from mild asymptomatic illness to fatal dengue hemorrhagic fever(DHF) and dengue shock syndrome(DSS) $)^{2}$.Fever, headache,

myalgia/arthralgia, nausea, vomiting and maculo-papular rashes are the clinical symptoms of classic dengue fever presentation ${ }^{4}$.Other infections like malaria, typhoid, and leptospirosis can mimic dengue and laboratory investigations are essential for an early definite clinical diagnosis ${ }^{6}$.

The diagnoses can be done with different biomarkers. They include isolation of virus in culture or mosquitoes or detection of viral genomic RNA, capture and detection of viral products (NS1 protein) or the host immune response to viral infection (measurement of virus specific immunoglobulin $\mathrm{M}$ and $\mathrm{G}$ (IgM and $\operatorname{IgG})^{10}$.A significant rising IgM levels 3-5 day after the onset of symptoms shows a primary infection.This can persist for 1-3 months. In secondary infection there will be elevated levels of $\mathrm{IgG}$ at 6-15 days of symptoms and IgM can also be detected in secondary infection ${ }^{11}$. As per the World Health Organization(WHO)dengue case definition in acute febrile illness 2 blood samples to be collected. First sample in 1-5 days of onset of symptoms and second sample 6-14 days after the onset of symptoms during the convalescent phase ${ }^{12}$. This study was done for the evaluation of rapid card tests and capture ELISA tests.

\section{Materials and Methods}

The study was conducted in the department of microbiology laboratory, KS HEGDE MEDICAL ACADEMY, with the blood samples collected from the patients of febrile illness(0-15 days) with clinical diagnosis of dengue (Sample size of 50) from the period of October 2015 to April 2017.

\section{Method of processing}

\section{Collection and transport of samples}

Blood samples were collected in red-capped vacutainer with all aseptic preparations. Samples werecentrifuged and plasma separated. Samples were processed according to the manufacturers instruction. Those samples not processed within 6 hours were refrigerated at 2-8 degree centigrade and were processed within 3 days. From each patient 2 blood samples (Sample 1 between 1-5 days of clinical symptoms and sample 2 between 15 21 days of clinical Symptoms) were collected.

\section{Dengue day 1 rapid test}

Dengue Day 1 Test kit contains two devices; one device for Dengue NS1 antigen detection and other device is for the differential detection of Dengue IgM / IgG antibodies in human serum/ plasma. Dengue IgM/IgG test device is containing three lines; Control line "C", IgM test line "M" and IgG test line"G". IgM and IgG test line test line are coated with anti-human $\operatorname{IgM}$ monoclonal antibodies and anti-human IgG monoclonal antibodies respectively.

Test was done as per the manufacturers instruction. The results were read after 20 
minutes (Positive results will appear as early as 2-10 minutes. Negative results were confirmed after 20 minutes only).

\section{ELISA tests}

\section{Specimen processing}

\section{Frozen sample}

Dengue ELISA tests will give the best performance if tests are done with fresh samples that have not been frozen and thawed. In this study few samples were run fresh and the remaining was kept in the refrigerator at 28 degree. Kit \& its components were stored at $2-8^{\circ} \mathrm{C}$. (Expiry date on the kit indicates the date beyond which kit should not be used).

\section{Test principle}

(A) NS1Ag MICROLISAis a solid phase enzyme linked immunoassay (ELISA) based on the "Direct Sandwich" principle. (B) DENGUE IgM MICROLISAis an enzyme immunoassay based on "MAC CaptureELISA". (C) DENGUE IgG MICROLISAis an enzyme immunoassay based on "GAC-Capture ELISA". Testswere done as per the manufacturers instruction.

\section{Results and Discussion}

Patients admitted in the hospital with a clinical history suggestive of dengue fever and a positive dengue test (NS1 Ag / IgM/ IgG) from the period of October 2015-April 2017 were included Initial study population was 132 dengue positive cases. For the fulfillment criteria of this study 2 blood samples had to be collected from each patient. First blood sample was collected from all the 132 patients. Despite all efforts for adequate sample collection, 11 serum samples received were inadequate to proceed with ELISA tests. From the remaining 121 patients only 67 patients were attending for a follow up and second sample were collected only from these patients only. So the final numbers were restricted to 67 cases though the sample size in the study criteria was only 50. First sample was collected between 1-5 days of the onset of symptoms and the second sample 15-21 days later. Relevant clinical history was collected from the patient or patient party and there after serum sample was collected. For all these patients both and Rapid and ELISA tests were done.

\section{Age distribution}

$27(40.3 \%)$ patients out of the 67 cases were in the age group of $31-45$ followed by 24 cases $(35.8 \%)$ in the age group of 46-60yrs. Only 4 $(6 \%)$ cases were seen in>15 yrs age and $3(4.5 \%)$ patients were>61 yrs. In 16-30 yrs age group 9 patients (13.4\%)were having dengue fever in our study.

In another study by Solanke et al showed $81.9 \%(127 / 155)$ of their dengue positivecases were in the age group of 0-20 years, followed by the age group of 21-40 years ${ }^{14}$.

\section{Sex distribution}

Maleswere more affected with dengue fever in this study, $36(53.7 \%)$ of 67 cases. Females were $31(46.3 \%)$. In astudy by Jigna karia et al, out of the 78 dengue positive cases $53(67.94 \%)$ were males and $25(32.05 \%)$ were females ${ }^{44}$.

In the study by Tabasum begum et al conducted in Mysore, which is belonging to the state of Karnataka in the year 2013, showed $104(66.24 \%)$ male cases and 53 $(33.76 \%)$ females out of the 157 dengue fever cases $^{36}$.

\section{Clinical symptoms}

In our study fever was the commonest presenting symptom in $64(95.5 \%)$.Other 
symptoms like headache in $60(89.6 \%)$,myalgia /arthralgia in $55(82.1 \%)$ ,retro-orbital pain in $27(40.3 \%)$. Less common symptoms like abdominal pain was seen in 15 $(22.4 \%)$ nausea /vomiting was present in 10 $(14.9 \%)$ and skin rash was seen in $8(11.9 \%)$ cases.Dengue Hemorrhagic Fever (DHF) was present in only 1 case $(1.5 \%)$.

In a study by Gopalakrishna S and Mohan K, from Bangalore Karnataka in the year 2012 during an epidemic, all the patients were presenting with fever(100\%).Other symptoms were headache $(95.7 \%)$, myalgia/arthralgia (92.1\%), retro-orbital pain (78\%), hemorrhagic manifestation $(29.7 \%)^{47}$. Most of the findings in this study favor our study findings.

\section{Laboratory findings}

In this study thrombocytopenia was present in 27(40.3\%) patients. Thrombocytopenia (Platelets $<50,000 / \mu \mathrm{l}$ ) is a very significant indicator in dengue fever. Another findings were leukopenia (WBC < 4000/ $\mu \mathrm{l}$ ) in 8 $(11.9 \%)$ cases, SGPT $>55 \mathrm{u} / 1$ in $5(7.4 \%)$, SGOT $>45 \mathrm{u} / \mathrm{l}$ in $7(10.4 \%)$ and Total bilirubin $>2 \mathrm{mg} / \mathrm{dl}$ in $1(1.5 \%)$. Increased Hematocrit values were seen in $12(17.9 \%)$ cases. In our study DHF was diagnosed in, 1 (1.5\%) patient only. No cases of DSS or death were reported in our study.In a study by Aswinikumar et al,8\%showed DHF and 7.3\% with DSS and 3 deaths were also reported ${ }^{47}$.

In case of DSS there will be severe plasma leakage, which can cause shock or accumulation of fluid and can cause a respiratory distress, bleeding and organ damage (due to metabolic acidosis). So DSS patients should be closely monitored. Management protocols of DSS patients are given in WHO handbook for the management of Dengue ${ }^{10}$.

\section{Serological tests}

NS1 Ag Detection by Dengue day 1 (Rapid test) and ELISA tests

Non-structural protein1(NS1) is a glycoprotein, produced in secretory and membrane associated forms by the virus ${ }^{48}$.The NS1Ag levels can be detected by immunochromatography and sandwich Elisa.In the diagnosis of acute dengue infection NS1Ag detection represented a newer approach ${ }^{49}$.

Acute serum sample (Sample 1: Between 15 days)

\section{Rapid test}

In this study out of 67 dengue positive patients, Rapid test showed 54 NS1Ag positive and 13 NS1Ag negative.

NS1 MICROLISA-ELISA was showing 54 NS1Ag positive and 13 negative. Comparative evaluation of the rapid test was done considering ELISA as the gold standard. Rapid test was showing $98.1 \%$ sensitivity, $92,3 \%$ specificity with PPV $98.1 \%$ and NPV92.3\% and diagnostic accuracy of $97.01 \%$.

\section{Convalescent serum sample (Second sample: between 15-21 days)}

RAPID TEST-The second sample showed $41(61.19 \%)$ NS1Ag positive cases by Dengue day 1 test and 26(38.8\%) negative cases.

ELISA test was giving 40(59.70\%), positive case and 27(40.29\%) negative. However 1 patient with NS1Ag negative report detected by rapid test was giving NS1Ag positive report in capture ELISA test. This can be due to a false negative report. At the same time one case with NS1Ag positive report given by 
Rapid test was giving report by capture Elisa. The sensitivity and specificity of Rapid test was $97.5 \%$ and $92.6 \%$ with a PPV of $95.1 \%$ and NPV of $96.2 \%$ with a diagnostic accuracy of $95.52 \%$.

In a study done in Maharashtra India, NS1Ag immunochromatographic test was compared with capture ELISA. It was showing $90.11 \%$ sensitivity and $98.45 \%$ specificity with a PPV of $98.15 \%$ and a NPV of $91.57 \%{ }^{51}$.This is similar to the findings in our study. However we cant exclude dengue infection immediately after a negative NS1Ag report by rapid test. These reports along with antibody detection assays will provide a higher diagnostic yield ${ }^{34}$.

\section{IgM Antibody (Acute serum sample)}

dengue day 1 test and MAC ELISA test: A comparative evaluation

In the Rapid test for detecting $\operatorname{IgM}$ antibody from the 67 acute serum samples 9(13.4\%) were positive for $\operatorname{IgM}$. Out of this $9 \operatorname{IgM}$ positive cases 3 were positive for NS1Ag also. $5 \mathrm{IgM}$ positive cases were negative for NS1Ag .One patient with NS1Ag positivity was found to be positive for both IgM and IgG.

\section{MAC ELISA}

In the MAC ELISA test for detecting IgM antibody from the 67 acute serum samples 9 (13.43\%) were positive for IgM antibody. Out of this $9 \mathrm{IgM}$ positive cases 4 cases were having positive NS1Ag also. 5 IgM positive cases were NS1Ag negative. MAC ELISA test has been taken as the gold standard for a comparative evaluation.

Dengue DAY 1 test for was showing at 88.9 $\%$ and a specificity of $98.3 \%$. The PPV was $88.9 \%$ and NPV at $98.3 \%$ with a diagnostic accuracy of $97.01 \%$.

\section{Convalescent serum (Day 15-21)}

Dengue day 1 test- In the Rapid test for detecting $\operatorname{IgM}$ antibody from the convalescent serum samples $27(40.3 \%)$ were positive for IgM antibody, 10 cases from this were having positive NS1Ag also. $8 \mathrm{IgM}$ positive cases were seenwith NS1Ag negative patients. 9 patients were found to be positive for both IgM and $\mathrm{IgG}$, out of this 1 patient was NS1 Ag positive while 8 were NS1Ag negative.

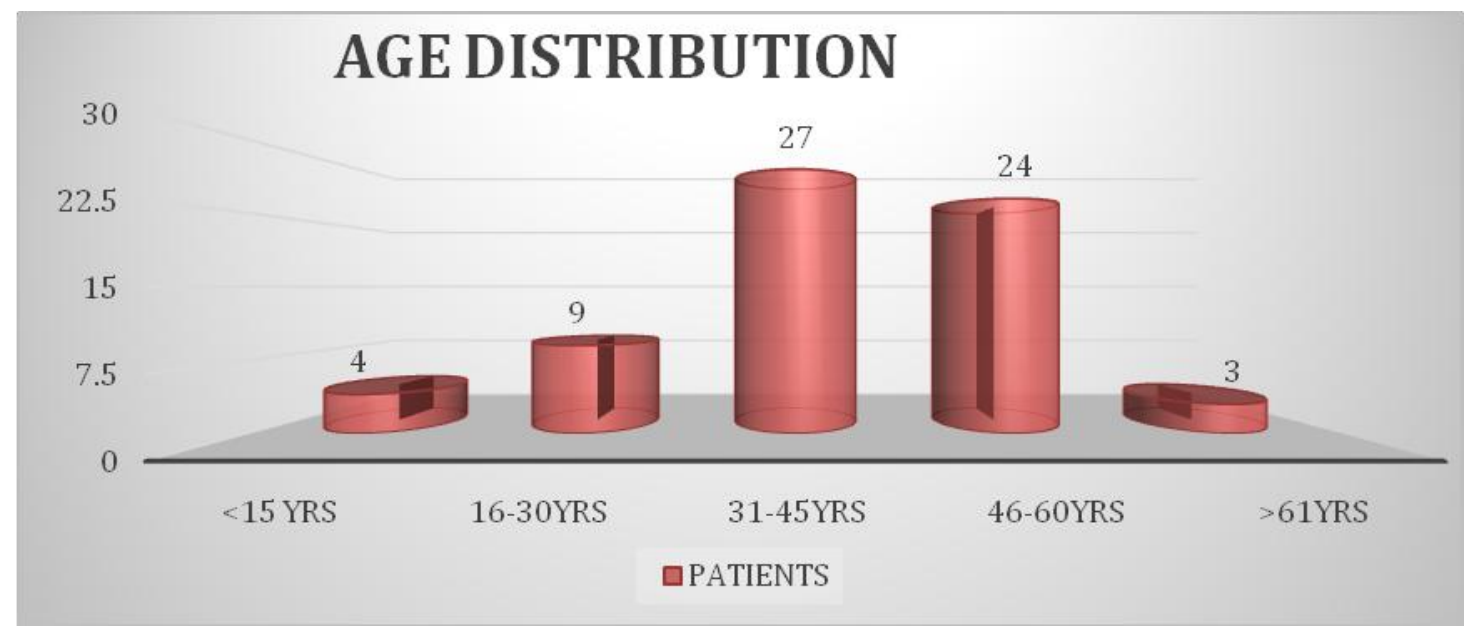



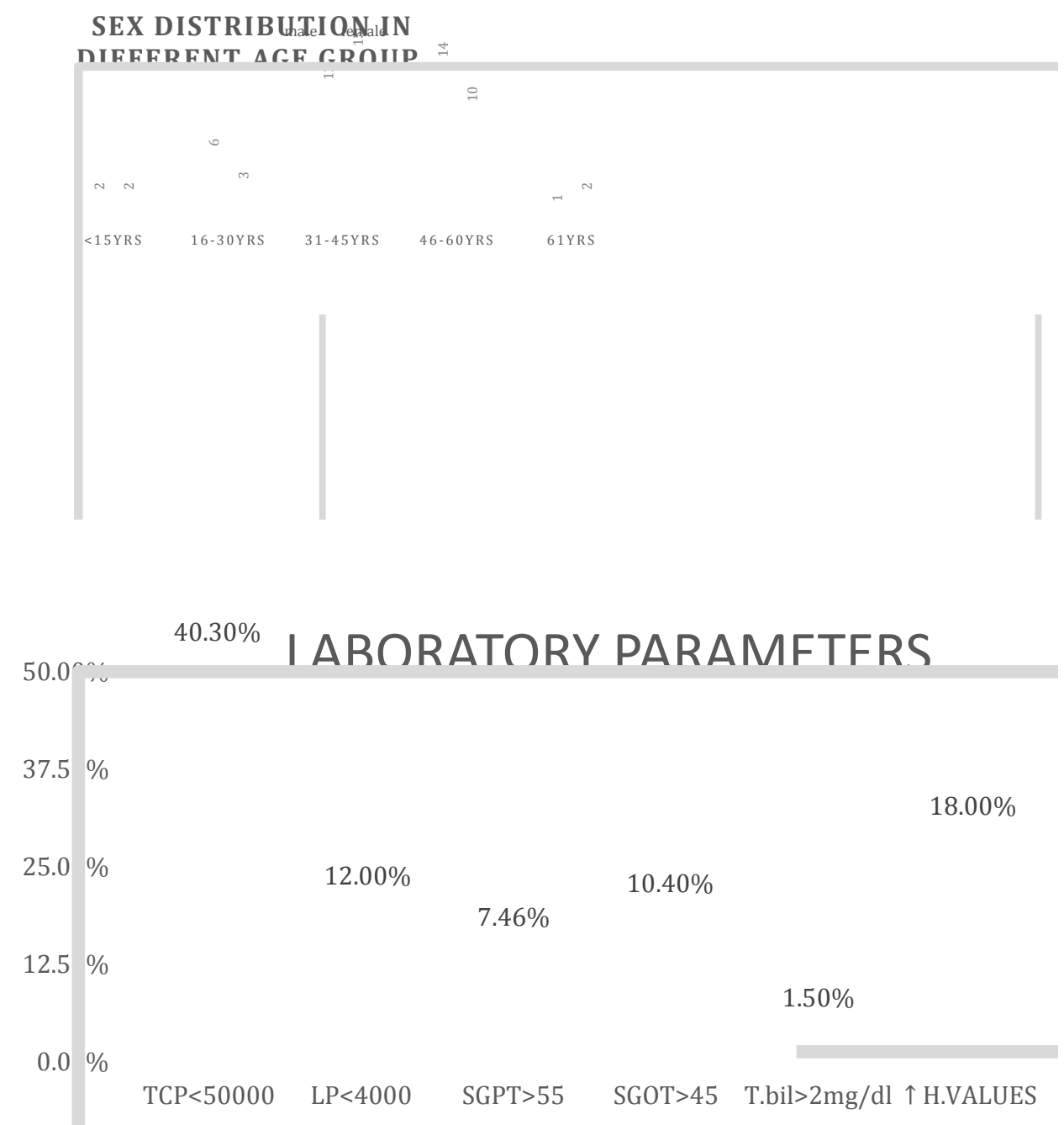

Abbereviations; TCP; thrombocytopenia, LP;leukopenia, SGPT;alanine aminotransferase(ALT), SGOT; aspartate amino tranferase(AST), T.Bil; Total bilerubin, $\uparrow$ H.value; increased hematocrit values

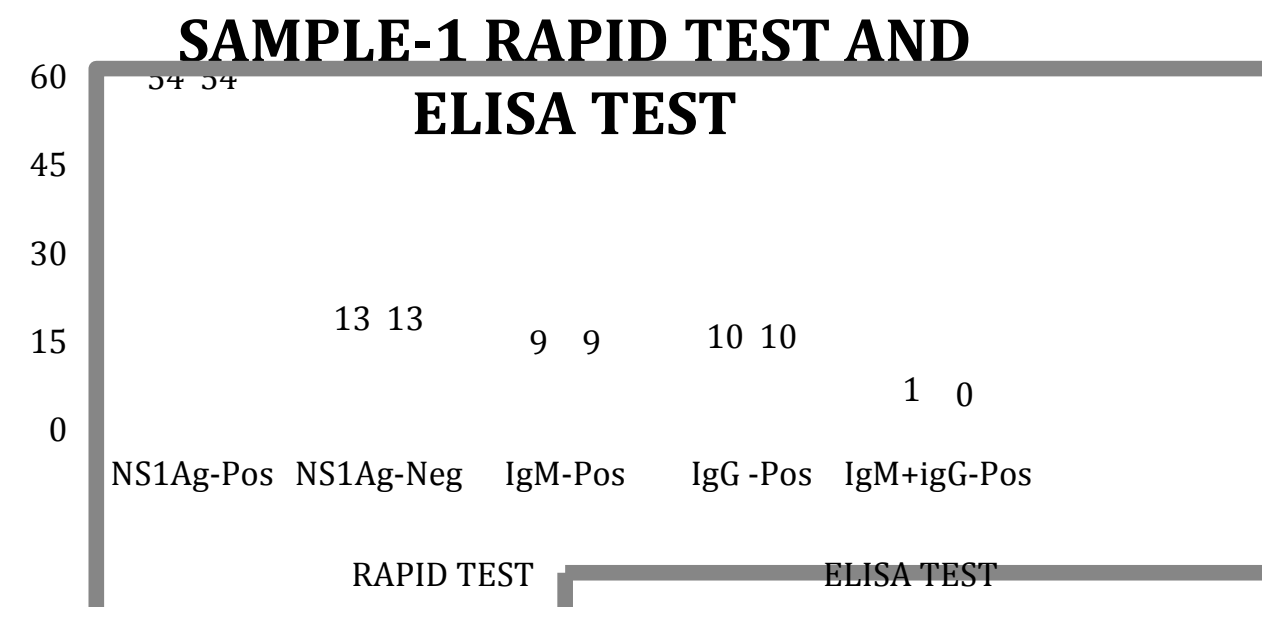



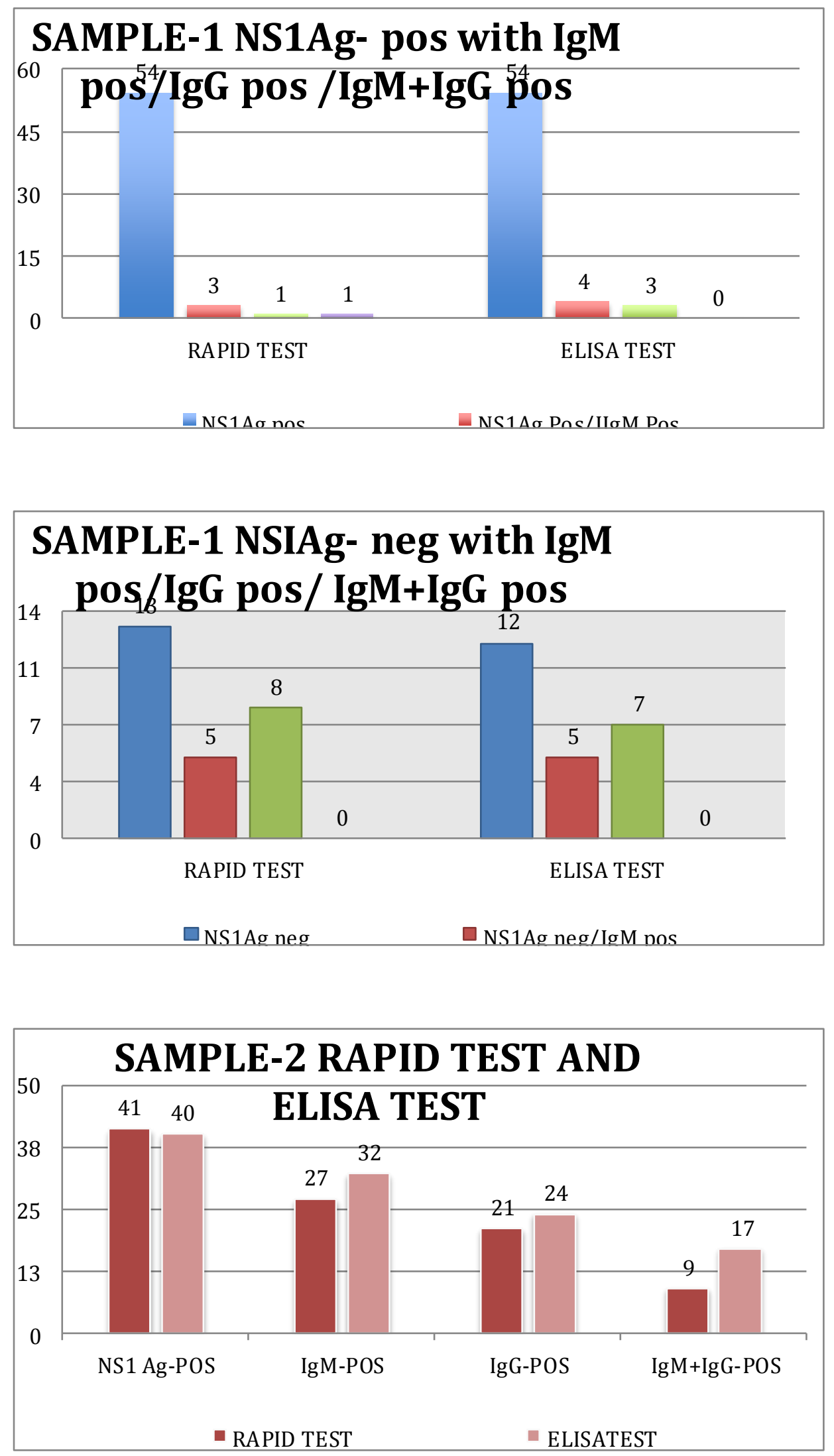

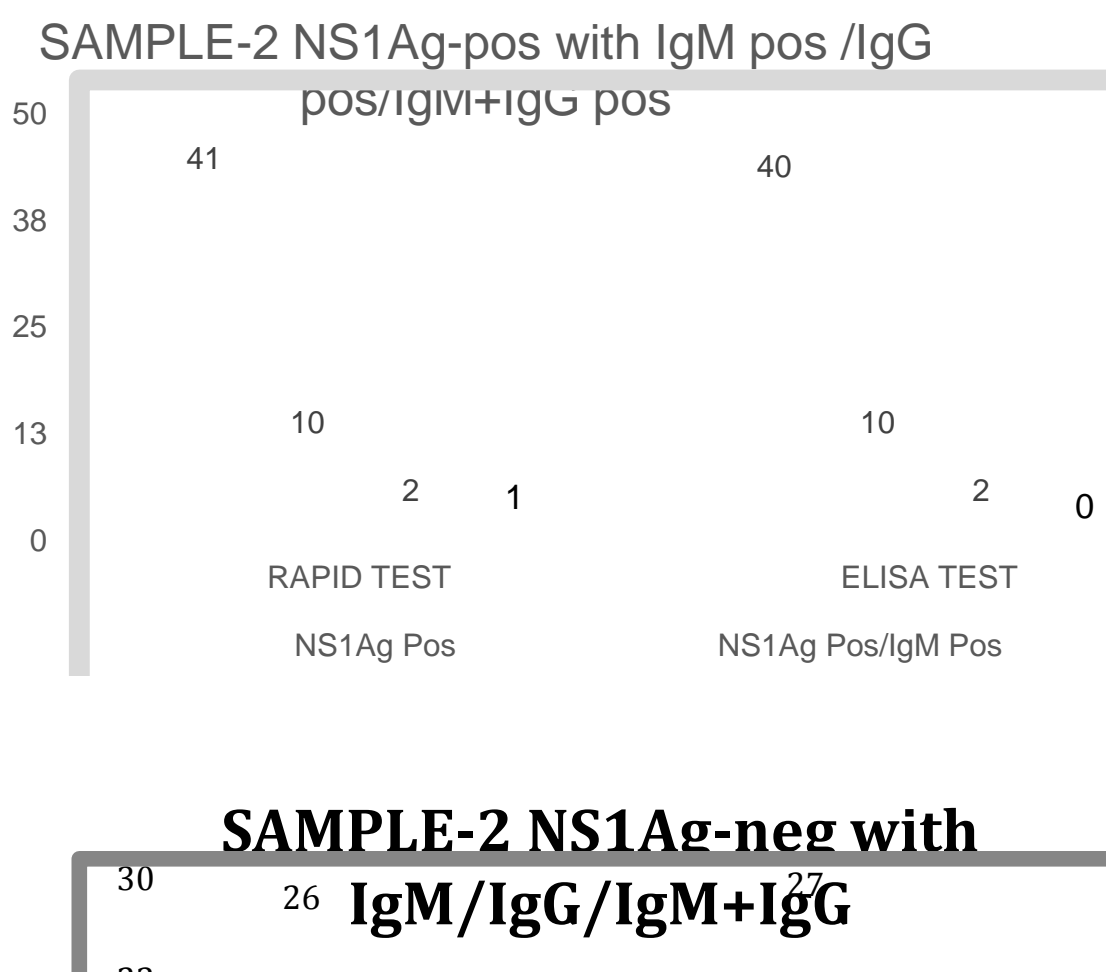

23

15

$\begin{array}{llll}8 & 10 & 8\end{array}$

8

0

RAPIDTEST

NS1Ag Neg

NS1Ag neg/IgG Pos $4 \quad 6$

ELISA TEST

NS1Ag Neg/IgM Pos NS1Ag Neg//IgM + IgG Pos

\section{MAC ELISA-A comparative evaluation}

In the MAC ELISA test for IgM antibody from the convalescent serum samples 31 (46.2\%) of 67 cases were found positive for IgM antibody. Out of these 31 cases 10 cases were positive for NS1Ag. 4 NS1Ag negative patients were IgM positive. At the same time 17 NS1Ag negative patients were having both $\operatorname{IgM}$ and $\operatorname{IgG}$ positivity.

The Dengue Day 1 test was found to have a sensitivity of $83.9 \%$ and a specificity of $97.2 \%$ with a PPV of $96.3 \%$ and a NPVof
$87.5 \%$. The diagnostic accuracy was $91.04 \%$.

In some studies increased sensitivity of the RAPID test devices were seen in primary infection comparing to secondary infection. During the time period of 0-3 days; sensitivity of IgM rapid tests increased. Appearance of IgM antibody and the absence of IgG indicate a primary infection. At the same time presence of $\mathrm{IgG}$ antibody is indicative of secondary infection. Moreover the duo cassettes have a draw back of misdiagnosing primary as secondary dengue infection ${ }^{13}$. 


\section{IgG Antibody}

\section{Dengue day 1 Rapid test and GAC ELISA a comparative evaluation:}

\section{Acute serum sample: Rapid day 1 test}

From the 67 acute serum samples 10 (14.9\%), were positive for $\operatorname{IgG}$ antibody. Out of the 10 IgG positive cases; 1 case was positive for NS1Ag showing a probable recent infection. But presence of IgG antibodies in the early stage could be due to a past infection with another strain.1 patient with NS1Ag positivity was showing $\operatorname{IgM}$ and $\mathrm{IgG}$ antibody $.8 \mathrm{IgG}$ positive cases were found to be negative for NS1Ag.

\section{Acute serum sample: GAC ELISA a comparative evaluation}

In the GAC ELISA test for IgG antibody from the 67 acute serum samples 10 (14.9\%) patients were positive for $\operatorname{IgG}$ antibodies. Out of this $10 \mathrm{IgG}$ positive 3 were $\mathrm{NS} 1 \mathrm{Ag}$ positive while 7 cases were negative for NS1Ag.

Dengue day1 test showed 90\% sensitivityand 98.2\% specificity, with a PPV of $90 \%$ and NPV of $98.2 \%$ with a diagnostic accuracy of $97.01 \%$.

\section{Convalescent serum: DengueDAY 1 Test}

In the Rapid test for detecting IgG antibody from the convalescent serum samples 21 $(31.3 \%)$ patients were positive for $\operatorname{IgG}$ antibody. Out of these $21 \mathrm{IgG}$ positive cases, 2 cases were found to be positive for NS1Ag also. $10 \mathrm{IgG}$ positive cases were from NS1 Ag negative patients and 1 patient with NS1Ag positivity was showing $\operatorname{IgM}$ and $\operatorname{IgG}$ antibody positivity. At the same time $8 \mathrm{NS} 1 \mathrm{Ag}$ negative patients were found to be positive for both $\operatorname{IgM}$ and $\operatorname{IgG}$.

\section{Convalescent sera GAC ELISA a comparative evaluation}

$25(35.8 \%)$ patients were positive for $\mathrm{IgG}$ antibody. Out of this only 2 cases were positive for NS1Ag. 6 IgG positive cases were found to be negative for NS1Ag. At the same time $17 \mathrm{NS} 1 \mathrm{Ag}$ negative patients was found to be positive for both IgM and IgG on ELISA.This test showed a sensitivity of $80 \%$ with a specificity of $97.6 \%$. The PPV was $95.2 \%$ with a NPV of $89.1 \%$. The diagnostic accuracy was $91.04 \%$.

In a multi country study by Subhamoy Pal claimed that IgM and IgG with NS1Ag were giving a higher overall sensitivity for rapid test kits. They claimed that the sensitivity increased upto $93.5 \%$ in day $4-7,98.6 \%$ in day 8-14 and $93.9 \%$ from day 15 . In the same study MAC Elisa and GAC Elisa sensitivity reached upto $100 \%$ for the samples collected between the days 8-14. The specificity of $\operatorname{IgM}$ Elisa, was $88.1 \%$ in these samples, IgG specificity was $88.4 \%$. IgG Elisa was showing a lower sensitivity range in all serotypes. In secondary infection IgG showed still lower sensitivity $(69.6 \%)$. In contrast to this in primary and secondary infection IgM was having the similar sensitivity ${ }^{13}$. This was similar to our findings.

Dengue hemorrhagic fever is characterized by increase in vascular permeability and coagulopathy. In the acute phase of Dengue disease NS1protein is highly conserved and circulated in high levels in all dengue serotypes. Development of DHF is in correlation with this ${ }^{41}$. In this study there was one case of DHF.This case was showing NS1Ag positivity in acute serum sample by both Day 1 test and ELISA. This patient was showing thrombocytopenia.

In conclusion, rapid tests for dengue diagnoses, which are based on immune- 
chromatography, are mainly used in common clinical practice. Rapid tests are easy to use, cheap, need less expertise and another important factor behind is less turn over time. Performance of this RDT kits varies. Dengue fever is common in tropical regions where temperature and humidity is high. So storage temperature is a factor in kit performance. For easy diagnoses in a resource limited setups RDTs will be useful. Sensitivity and specificity of rapid kits still remains as a major issue and standardized approaches should be implemented while performing the diagnostic assessments. However ELISA has got a better sensitivity and specificity especially in secondary infections.

Elisa can be used in hospital when there is an outbreak of dengue as the format enables testing of multiple samples. At the same time it is a huge logistic burden for the laboratory when the time and training requirements are considered. The low specificity of the rapid tests will necessitate confirmatory test for all the RDT positive samples with ELISA tests.

\section{Acknowledgement}

I express my deep gratitude to my respected teacher and guide, Dr. Vimal Kumar Karnaker, Professor, Dr. Rekha Rai, Professor and Head, Department of Microbiology, K.S. Hegde Medical Academy, for their constant guide and encouragement and to Dr. P.S.Prakash, Dean, K.S. Hegde Medical Academy for the approval of this study. I am thankful to my teachers, colleagues all the non- teaching staff in the department of Microbiology for their kind support.

\section{References}

1. Costa RL, Voloch CM, Schrago CG. Comparative evolutionary epidemiology of dengue virus serotypes infection genetics and evolution. Infect Genet
Evol. 2012 Mar; 12(2): 309-14.

2. Gupta N, Srivastava S, Jain A, Chaturvedi UC. Dengue in India. Indian J Med Res. 2012 Sep; 136(3): 373-90.

3. Maria G Guzman Gustavo Kauri. Dengue diagnosis advances and challenges. International journal of infectious diseases. 8(2004) 69-80.

4. Sangita Kamath, Neeraj Jain, Satish Gupta, A C Jha and B S Rao. Dengue Epidemic in Jamshedpur-Tata Main Hospital (TMH) Experience. J Trop Dis 2015, 3:2.

5. Vikram Khan, Daolatsinh Zala, Sandeep Sanghvi H.C. Srivastavaand V K. Das. Occurrence of Dengue Cases in Silvassa, Dadra Nagar Haveli (Union Territory), India. J Trop Dis 2016, 4:5.

6. Damodar T, Dias M, Mani R, Shilpa KA, Anand AM, Ravi V, Tiewsoh J Clinical and laboratory profile of dengue viral infections in and around Mangalore India. Indian J Med Microbiol 2017; 35:256-61.

7. Irani Ratnam, Karin Leder, Jim Black, Mand Joseph Torresi, Dengue Fever and International Travel. Journal of Travel Medicine 2013; 20 (6): 384-393.

8. Louis Jose Dzouza, Lais Bastos, Pessanha,Laura, Curvalho Mansur. Comparison of clinical and laboratory characteristics between children and adults with dengue. Braz $\mathrm{j}$ infect dis. 2013; 17(1): 27-31.

9. K Jayashree G, C Manasa P, Pallavi G, V Manjunath. Evaluation of Platelets as Predictive Parameters in Dengue Fever. Indian Journal of Hematology and Blood Transfusion. July-Sept 2011; 27(3): 12730.

10. Muller DA, Depelsenaire AC. Young PR. Clinical and Laboratory Diagnosis of Dengue Virus Infection. J Infect Dis. 2017; 215(2): 89-95

11. Scott R Fry, Michelle Meyer, Mathew G Sample. The Diagnostic Sensitivity of 
Dengue Rapid Test Assays Is Significantly enhanced by Combined Antigen and antibody testing approach. PLoS Neglected Tropical Diseases. June 2011: 5 (6).

12. Hunsperger E A, Muñoz-Jordán J, Beltran M. Performance of Dengue Diagnostic Tests in a Single Specimen Diagnostic Algorithm. Infect Dis 2016 Sep 15; 214(6): 836-44.

13. Pal S, Dauner A L, Valks A, Forshey B M. Multicounty prospective clinical evaluation of two enzyme-linked immunosorbent assays and two rapid diagnostic tests for diagnosing dengue fever. J Clin Microbiol. 2015 Apr; 53(4): 1092-102.

14. Vaishali N Solanke Mohan G Karmarker Preeti R Mehta. Early dengue diagnosis: Role of rapid NS1 antigen, NS1 early ELISA, and PCR assay. Trop J Med Res 2015; 18:95-9.

15. Hunsperger E A, Sharp TM, Lalita P. Use of a Rapid Test for Diagnosis of Dengue during Suspected Dengue Outbreaks in Resource Limited Regions. J Clin Microbiol. 2016 Aug; 54(8): 2090-5.

16. Pal S, Dauner AL, Mitra I, Forshey BM, Garcia P. Evaluation of Dengue NS1 Antigen Rapid Tests and ELISA Kits Using Clinical Samples. PLoS One. 2014 Nov 20; 9 (11).

17. Koo C, Nasir A, Hapuarachchi H C. Evolution and heterogeneity of multiple serotypes of Dengue virus in Pakistan, 2006-2011. Virol J. 2013 Sep 4; 10:275.

18. Arvind Neralwar, Bimala Banjare, Barapatre R. Detection of NS1 antigen, IgM antibody for the diagnosis of dengue infection in patients with acute febrile illness. Int J Res Med Sci. 2015 Oct; 3(10): 2826-2830.

19. Gupta E, Ballani N. Current perspectives on the spread of dengue in India Infect Drug Resist. 2014 Dec 11; 7:337-42.

20. Kumar NP, Jayakumar PR, George K.
Genetic characterization of dengue viruses prevalent in Kerala State, India $\mathrm{K}$ J Med Microbiol. 2013 Apr; 62(4): 54552.

21. Moi ML, Omatsu T, Tajima S, Lim CK. Detection of dengue virus nonstructural protein 1 (NS1) by using ELISA as a useful laboratory diagnostic method for dengue virus infection of international travelers. J Travel Med. 2013 May-Jun; 20(3): 185-93.

22. Shrivastava A, Dash PK, Tripathi NK .Evaluation of a commercial Dengue NS1 enzyme-linked immunosorbent assay for early diagnosis of dengue infection. Indian J Med Microbiol. 2011 Jan-Mar; 29(1): 51.

23. Ahmed NH, Broor S. Comparison of NS1 antigen detection ELISA, real time RTPCR and virus isolation for rapid diagnosis of dengue infection in acute phase. J Vector Borne Dis. 2014 Sep; 51(3): 194-9.

24. Amarjeet Singh and Andrew W TaylorRobinson. Vector Control Interventions to Prevent Dengue: Current Situation and Strategies for Future Improvements to Management of Aedes in India. J Emerg Infect Dis 2:123.

25. Wahala WM, Silva AM. The Human Antibody Response to Dengue Virus Infection Viruses. 2011 Dec; 3(12): 2374-95

26. Cecilia D, Kakade MB, Bhagat AB, Vallentyne J, Singh A, Patil JA, Todkar SM Detection of dengue-4 virus in Pune, Western India after an absence of 30 years--its association with two severe cases. Virol J. 2011 Feb 1; 8:46.

27. Nguyen Tien Huy, Huynh Trung Trieu, Kenta Okamoto. Proteomic Profile of Circulating Immune Complexes in Dengue Infected Patients. J Trop Dis 2013 1(2): 109.

28. Kaihatsu K, Harada E, MatsumuraH, Takenaka A. Future Perspective of 
Nucleic Acid-based Detection of Dengue Virus and its Serotypes. J Antivir Antiretrovir . 2016, 8:2.

29. Chuang YC, Wang SY, Lin YS, Chen HR, Yeh TM. Re-evaluation of the pathogenic roles of nonstructural protein 1 and its antibodies during dengue virus infection. J Biomed Sci. 2013 Jun 27; 20:42.

30. Duong V, Ly S, Lorn Try P, Tuiskunen A. Clinical and virological factors influencing the performance of a NS1 antigen-capture assay and potential use as a marker of dengue disease severity. PLoS Negl Trop Dis. 2011 Jul; 5(7).

31. Madura Adikari. Charith Perera, Mihika Fernando. Prevalence of Hypocalcaemia and Its Potential Value as a Biochemical Marker in Patients with Severe Dengue Infection J Trop Dis (2015) 4:2; 188

32. Vajpayee M, Singh UB, Seth P, Broor Comparative evaluation of various commercial assays for diagnosis of dengue fever. Southeast Asian J Trop Med Public Health. 2001 Sep; 32 (3) 472-5.

33. Bessoff K, Phoutrides E, Delorey M, Acosta LN, Hunsperger E.Utility of a commercial nonstructural protein 1 antigen capture kit as a dengue virus diagnostic tool. Clin Vaccine Immunol. 2010 Jun; 17(6): 949-53.

34. Huits R, Soentjens P, Maniewski-Kelner U, Theunissen C, Van Den Broucke S, Florence E, Clerinx J, Clinical Utility of the Nonstructural 1 Antigen Rapid Diagnostic Test in the Management of Dengue in Returning Travelers With Fever. Open Forum Infect Dis. 2017 Jan 9; 4(1).

35. N S Chithambaram, Kushal D Shah. To evaluate the role of NS1antigen for early detection of dengue fever. $\mathrm{J}$ evolution of med and dent scie 2014 Dec 18: 3(71).

36. Tabasum Begum M Dr. Sumana MN Dr. Basavana Gowdappa H. Evaluation of
Rapid ICT in comparison with MACELISA in diagnosis of Dengue fever at a tertiary care hospital, South India. IJPSI. December 2014: 3(12) 11-16

37. Prince HE, Yeh C, Lapé-Nixon M. Utility of $\mathrm{IgM} / \mathrm{IgG}$ ratio and $\mathrm{IgG}$ avidity for distinguishing primary and secondary dengue virus infections using sera collected more than 30 days after disease onset. Clin Vaccine Immunol. 2011 Nov; 18(11): 1951-6.

38. Zhang $\mathrm{H}$, Li W, Wang J, Peng H, Che X, Chen X, Zhou YNS1-based tests with diagnostic utility for confirming dengue infection: a meta-analysis. Int $\mathrm{J}$ Infect Dis. 2014 Sep; 26:57-66.

39. Rubens Costa Lima J, Rouquayrol MZ, Monteiro Callado MR, Florindo Guedes MI, Pessoa C.Interpretation of the presence of $\operatorname{IgM}$ and $\operatorname{IgG}$ antibodies in a rapid test for dengue: analysis of dengue antibody prevalence in Fortaleza City in the 20th year of the epidemic. Rev Soc Bras Med Trop. 2012 Mar-Apr; 45(2): 163-7.

40. Changal KH, Raina AH, Raina A, Raina M, Bashir R, Latief M, Mir T, Changal QHDifferentiating secondary from primary dengue using $\operatorname{IgG}$ to $\operatorname{IgM}$ ratio in early dengue: an observational hospital based clinico-serological study from North India.. Infect Dis. 2016 Nov 28; 16(1): 715.

41. Vickers IE, Harvey KM, Brown MG, Nelson K, DuCasse MB, Lindo JFThe performance of the SD BIOLINE Dengue DUO ${ }^{\circledR}$ rapid immunochromatographic test kit for the detection of NS1 antigen, $\mathrm{IgM}$ and $\mathrm{IgG}$ antibodies during a Dengue type 1 epidemic in Jamaica.J Biomed Sci. $2015 \mathrm{Jul} 16 ; 22: 55$.

42. Pothapregada S, Kamalakannan B, Thulasingam M, Sampath S. is Reactive Dengue NS1Antigen Test a Warning Call for Hospital Admissions? J Clin Diagn Res. 2016 Apr; 10(4). 
43. R. Norrby. Outlook for a dengue vaccine Clin Microbiol Infect 2014; 20(5) 92-94.

44. Jigna Karia, Hetal Shah,Parul PatelJignasa Bhalodia, HitendraBhavsar, GaurishankarShrimali.Evaluation of commercial newer rapid test for detection of early acute dengue infection. Nati $j$ med res 2011; 1(2): 31-33.

45. Ronni Mol P Seroprevalence and Comparison of Rapid Immunochromatographic test and Elisa for the detection of NS1 antigen for the early diagnosis of Dengue fever. IJPBS Apr-Jun2015; 5 (2); 123-129.

46. Jyothi P, Metri BC.Correlation of serological markers and platelet count in the diagnosis of Dengue virus infection. Adv Biomed Res. 2015 Jan 30; 4:26.

47. Shashidhar Gopalakrishna Krithika Mohan. Correlation of Clinical, Serological and Radiological Features of Severe Dengue Fever Sch. J. App. Med. Sci., 2015; 3(3E): 1397-1399.

48. Datta S, Wattal C.Dengue NS1 antigen detection: a useful tool in early diagnosis of dengue virus infection. Indian $\mathrm{J}$ Med Microbiol. 2010 Apr-Jun; 28(2).

49. Dussart P, Petit L, Labeau B, Bremand L, Leduc A, Moua D. Evaluation of Two New Commercial Tests for the Diagnosis of Acute Dengue Virus Infection Using NS1 Antigen Detection in Human Serum Trop Dis. 2008 Aug 20; 2(8).

50. Chaterji S, Allen JC Jr, Chow A, Leo YS, Ooi EE.Evaluation of the NS1 rapid test and the WHO dengue classification schemes for use as bedside diagnosis of acute dengue fever in adults Am J Trop Med Hyg. 2011 Feb; 84(2): 224-8.

51. R. Mahesh Reddy, Kavita Sahai, Ajay Malik, S. Shoba \& Anurag Khera. Comparative Analysis of Rapid Dengue Testing and ELISA for NS1Antigen and IgM in Acute Dengue Infection Int.J.Curr.Microbiol.App.Sci (2016) 5(10): 931-93753.

52. Stuart D. Blacksell. Commercial Dengue Rapid Diagnostic Tests for Point-of-Care Application: Recent Evaluations and Future Needs? Journal of Biomedicine and Biotechnology. 2012 (2012).

53. Mitra S, Choudhari R, Nori H, Abhilash KP, Jayaseelan V, Abraham AM, Cherian AOComparative evaluation of validity and cost-benefit analysis of rapid diagnostic test (RDT) kits in diagnosis of Dengue infection using composite reference criteria: A cross-sectional study from South India.J Vector Borne Dis. 2016 Mar; 53(1): 30-6.

\section{How to cite this article:}

Rameena Anver, Vimalkumar Karnaker and Rekha Rai. 2018. Evaluation of the Rapid Card Test and Capture ELISA Tests in Diagnosis of Dengue Infection. Int.J.Curr.Microbiol.App.Sci. 7(07): 4049-4061. doi: https://doi.org/10.20546/ijcmas.2018.707.471 